\title{
Limnology of Surinsar lake, Jammu (J\&K State): Part I- Protozoa
}

\section{Slathia* and S. P. S. Dutta}

Department of Environmental Sciences, University of Jammu, Jammu -180006 (J\&K), INDIA

*Corresponding author. E-mail: dsenviron1@yahoo.co.in

\begin{abstract}
Zooplanktonic analysis of Surinsar lake, Jammu, during the year 2002-2004, has shown the presence of fifty one species of protozoans belonging to three classes viz. Sarcodina (32 spp), Ciliata (16 spp.) and Mastigophora (3 spp.). Quantitatively, during the year 2002-03, there was dominance of Sarcodina followed by Ciliata and Mastigophra. In the subsequent year viz. 2003-04, the order of quantitative dominance has been observed as Ciliata> Sarcodina and $>$ Mastigophora. Coefficient of correlation( $r$ ) of protozoans with various physico-chemical parameters has generally shown insignificant results. The calculated value of ANOVA of various protozoan classes was significant and the means of various classes differed significantly among themselves indicating their significant contribution to total protozoan population.
\end{abstract}

Keywords: Lake, Zooplankton, Protozoa, Correlation, Diversity Index

\section{INTRODUCTION}

Protozoans are cosmopolitan in distribution, predominantly microscopic, simplest in structure and the most abundant animals in the world in terms of number and biomass. These biological indicators(Katiyar and Belsare,1997; Xu et al., 2002; Lee et al., 2004 and Jiang and Shen, 2007), also play a significant role in biological wastewater treatment (Kotangale, 1995; Lee et al., 2004 and Jiang and Shen, 2007). Unlike water quality measurements, which only provide an instantaneous assessment of lake conditions, protozoan community can be used to identity past disturbances and toxic effects that are not readily detected by chemical means (Cairns and McCormick, 1993). Inspite of a major role played by these microscopic organisms in aquatic environment, protozoans are, probably, the least studied group of fresh water. The present detailed study is first in this direction from a subtropical lake- Surinsar, in Siwalik hills, Jammu.

\section{TOPOGRAPHY OF THE AREA}

Jammu region of $J \& K$ state has two important freshwater Siwalik lakes viz. Mansar and Surinsar. Surinsar- a subtropical fresh water lake is about $25 \mathrm{~km}$ North-east of Jammu city $(\mathrm{J} \& \mathrm{~K})$. It is situated at an elevation of 605 meters, in $75^{\circ} 02^{\prime} 303$ East Longitude and $32^{\circ} 46^{\prime} 303$ North Latitude. The spread of the lake varies from 27.14 ha (peak summer) to 27.92 ha (monsoons). The water level of the lake oscillates by $1.20 \mathrm{~m}$ and touches its peak during monsoon.

The littoral zone of lake has thick vegetation cover of emergent plants like Typha fatifolia, Phragmites sp., Ipomea cornea, Scirpus sp., floating vegetation like Nelumbium sp. and Nymphoides sp. and submerged vegetation comprising of Hydrilla verticillata, Najas graminae, Ceratophyllum demersum, Potamogeton crispus, $P$. nodosus, Chara fragalis, C. zeylanica, $C$. brauni, Nitella sp. etc. Fish fauna include Puntius conconius, P.ticto, P. chola, P. sophore, Rasbora rasbora, Channa punctatus and Heteropneustus fossilis. In addition, the lake supports two important species of turtles viz. Trionyx gangeticus and Lissemys punctata an endangered species (CITES and IUCN Red listed).

This lake is rich in micro-nutrients for which it is an attractive habitat, breeding and nursery ground for many migratory waterfowls. Important bird fauna(both native and migratory birds) of the lake includes Fulica atra atra(Coot), Gallinula chloropus indicus(Indian moorhen), Podiceps nigricollis, Podiceps cristatus(Great Crested Grebe), Podiceps ruficollis (Dabchick), Podiceps cristatus (Great crested Grebe), Aythya fuligula (Pochard), Netta rufina (Red Crested Pochard), Anas platyrhyncha platyrhyncha (Mallard or Wild Duck), Anas clypeata(Shoveller), Anas acuta(Pintail), Anas crecca(Common Teal), Phalacrocorax niger ( little Cormorant), P.corbo (large Cormorant), Egretta intermedia (Median egret), E. garzetta( little egret), Ardea alba (Large egret), Ardeola grayii (Pond heron), A. cinerea (Grey heron), Ceryle lugubris guttulata(Pied Kingfisher) and Alcedo atthis(Small blue kingfisher).

\section{MATERIALS AND METHODS}

Monthly planktonic samples, during the year 2002-03/ 2003-04, were collected by filtering 5 litres of water through a planktonic net (no. 25) in labeled plastic tubes. For live study and identification, separate samples were 
collected in a similar manner. Samples collected were fixed in $5 \%$ formaldehyde solution in labeled glass tubes, identified (Naidu, 1966; Nair et al., 1971; Dutta, 1983; Kudo, 1986 and Edmondson, 1992) and counted (n/l) by drop count method(Welch, 1948). Coefficient of correlation( $r$ ) and analysis of variance have been calculated following standard methods(Trivedy et al. ,1987 and Khan and Khanam, 2004).

\section{RESULTS AND DISCUSSION}

Fifty one species of protozoans, identified in lake Surinsar, have shown the qualitative dominance of class Sarcodina followed by Ciliata and Mastigophora (Tables 1 and 2). Sehgal (1980) observed two species viz. Difflugia sp. and Centropyxis sp. from Lake Surinsar, Jammu. Sharma(2000) analyzed zooplanktonic fauna of lake Mansar, Jammu and reported five species viz. Amphisiella oblonga, Centropyxis aculeate, Difflugia sp., Nuclearia sp. and Vorticella sp. Contrary to the present observation, Katiyar and Belsare(1997) noticed qualitative dominance of Mastigophora(24 spp.) followed by Ciliata( 8 spp.) and Sarcodina( 8 spp.), from Bhopal lakes, India and Kaushik and Saksena(1995) reported only class Sarcodina(6 spp.) from Gwalior .

Qualitative analysis of Protozoa has revealed the seasonal presence of various genera belonging to different classes. Earlier, Jhingran (1991) also documented seasonal planktonic association in tropical and sub-tropical climates. He pointed out that certain planktonic forms disappear at the specific periods and re-appear during others. Such temporary disappearances may be due to the fact that the species concerned either become too scarce or occur as spores or resting eggs etc. which are not easily detectable. Upon the return of the favourable conditions these plankters multiply and thus, increase in number.

Among the various genera of Mastigophora, during the first year, Euglena recorded its presence six times and Phacus and Chlamydomonas four times each (Table 1). In the subsequent year, Euglena is noticed nine times and Phacus and Chlamydomonas thrice each (Table 2). Seasonal qualitative analysis of class Mastigophora has revealed March and April high diversity with July, November, December and January disappearance, during the year 2002-03(Table 1). In the subsequent year viz. 2003-04, these recorded April and May increased diversity with complete disappearance during July, August and November (Table 2).

Among various genera of rhizopod protozoan, belonging to class Sarcodina, during the first year of study, only Arcella showed its perennial presence. Difflugia and Centopyxis recorded their presence ten times; Euglypha nine times; Nebela six times; Pyxidicula four times; Lesquereusia, Bullinula and Astrameoba thrice each and
Pareuglypha, Plagiopyxis and Ameoba twice each. Among the Actinopod protozoans, Actinophrys is seen only once(Table 1). During the subsequent year, among various genera of rhizopod protozoans, Arcella, Difflugia and Centropyxis remained perennial in the planktonic samples. Genus Euglypha was observed eleven times; Lesquereusia, ten times; Ameoba seven times; Plagiopyxis and Pyxidicula six times; Bullinula, Nebela and Astrameoba five times each and Pareuglypha once. Among the actinopod protozoans, Actinophrys made its record four times (Table 2).

Qualitative dominance of Difflugia over other genus of class Sarcodina, as observed in the present analysis of Surinsar lake water, is in accordance with the findings of Verma and Dalela(1974), Sharma and Rathore(2000) and Narayana and Somashekar(2002). Qualitative richness of various rhizopod species such asDifflugia, Centropyxis and Arcella in Surinsar lake water, may be due to their high tolerance to organic enrichment and is in agreement with the findings of Verma and Dalela (1974), Mishra and Saksena(1990), Srivastava et al.(1990), Kaushik and Saksena (1995) and Wetzel (2000).

An observation of Table 1 reveals April, May highest and December lowest qualitative diversity of class Sarcodina, during the first year. In the subsequent year, these recorded bimodal viz. March, April, September and October increase with March and April highest and November lowest diversity (Table 2).

Genus Euplotes, among various genera of class Ciliata, during the year 2002-03, showed its presence eight times; Paramecium seven times; Vorticella, Coleps and Stylonychia six times; Urocentrum and Carchesium four times, Halteria and Condylostoma thrice and Lionotus, Uronema and Homalozoon twice each(Table 1). During the subsequent year viz. 2003-04, genus Paramecium remained perennial; Urocentrum made its presence ten times; Vorticella and Euplotes nine times; Coleps eight times; Stylonychia and Carchesium six times; Halteria five times; Lionotus four times, Chilodonella, Uronema, Homalozoon and Condylostoma thrice each ( Table 2). Ciliates, qualitatively, during both the years, recorded March-May increase with a continuous decline from May onwards upto January. These observed April/April highest and January/ February lowest qualitative record. Seasonally, protozoan qualitative diversity remained high during summer (March-May), with April highest record, during both the years of study. These observed November, December and January/ November and December trough with lowest qualitative count during November (Tables 1 and 2).

Quantitatively, total protozoans, ranged between $59 \pm 53.18 \mathrm{n} / 1$ (December) to $1540 \pm 554.73 \mathrm{n} / 1$ (April) during the first year and $414 \pm 128.49 \mathrm{n} / \mathrm{l}$ (July) to $2538 \pm 783.40 \mathrm{n} / 1$ (April) in the subsequent year of study. 
Table 1. Mean monthly variations in Protozoa of Surinsar Lake, Jammu. ( Feb 2002-Jan 2003)

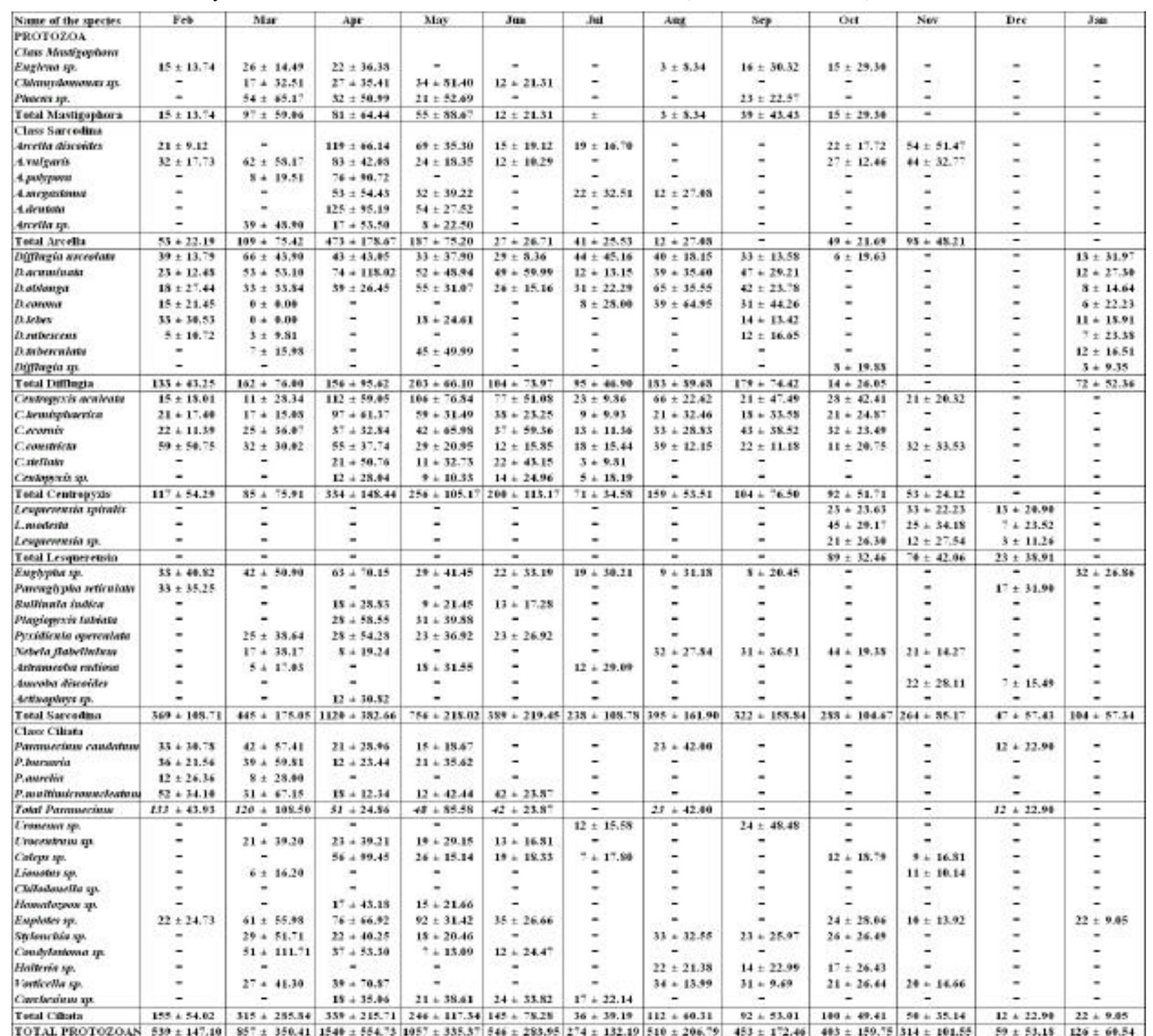

Quantitatively, the order of dominance of various classes of protozoans has been observed as Sarcodina $(47 \pm 57.43$ to $1120 \pm 382.66 \mathrm{n} / \mathrm{l})>$ Ciliata $(12 \pm 22.90$ to $339 \pm 215.71 \mathrm{n} / \mathrm{l})$ and $>$ Mastigophora (nil to $97 \pm 59.06 \mathrm{n} / \mathrm{l}$ ), during the year 2002-03 (Table I) . In the subsequent year viz. 2003-04, the order of quantitative dominance of various classes of protozoans has been noticed as Ciliata $(42 \pm 24.38$ to $1249 \pm 289.32 \mathrm{n} / \mathrm{l})>\operatorname{Sarcodina}(282 \pm 112.62$ to $1201 \pm 558.55 \mathrm{n} /$ 1) and > Mastigophora (nil to $88 \pm 71.15 \mathrm{n} / \mathrm{l}$ ) from Surinsar lake water (Table 2).

During the first year of study, quantitatively, the order of dominance of various genera, belonging to class Mastigophora, has been noticed as Phacus (nil to $54 \pm 65.17 \mathrm{n} / \mathrm{l}$ ) > Chlamydomonas (nil to $34 \pm 81.40 \mathrm{n} / \mathrm{l}$ ) and $>$ Euglena (nil to $26 \pm 14.49 \mathrm{n} / \mathrm{l}$ ). In the subsequent year, the order of quantitative dominance of various genera has been observed as Chlamydomonas (nil to $55 \pm 15.63 \mathrm{n} /$
1) $>$ Phacus (nil to $29 \pm 31.70 \mathrm{n} / \mathrm{l}$ ) >and > Euglena (nil to $29 \pm 31.70 \mathrm{n} / \mathrm{l})$

Seasonally, class Mastigophora, among various classes of Phylum Protozoa, showed bimodal viz March and September/April and September peaks, during the year 2002-03/2003-04. These recorded March/April highest and July, November and December/July, August and November absence(Table 1 and 2 and Fig. 1a).

The order of quantitative dominance of various genera belonging to Class Sarcodina, during the year 2002-03, has been observed as Arcella ( nil to $473 \pm 178.67 \mathrm{n} / \mathrm{l}$ ) > Centropyxis (nil to $334 \pm 148.44 \mathrm{n} / \mathrm{l}$ ) > Difflugia(nil to $203 \pm 66.10 \mathrm{n} / \mathrm{l}$ ) $>$ Lesquereusia (nil to $89 \pm 32.46 \mathrm{n} / \mathrm{l}$ )> Euglypha (nil to $63 \pm 70.15 \mathrm{n} / \mathrm{l})>$ Nebela (nil to $44 \pm 19.88 \mathrm{n} /$ 1) > Pareuglypha (nil to $33 \pm 35.25 \mathrm{n} / \mathrm{l}$ ) > Plagiopyxis (nil to $28 \pm 58.55 \mathrm{n} / 1$ ) and Pyxidicula (nil to $28 \pm 54.28 \mathrm{n} / \mathrm{l}$ ) $>$ Amoeba (nil to $22 \pm 28.11 \mathrm{n} / \mathrm{l}$ ) > Astramoeba (nil to 
Table 2. Mean monthly variations in Protozoa of Surinsar Lake, Jammu. (Feb 2003-Jan 2004)

\begin{tabular}{|c|c|c|c|c|c|c|c|c|c|c|c|c|}
\hline 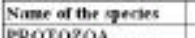 & Feb & Mise & 45 & May & Jain & Ind & Aag & Sep & OAt & Now & Dec & Jia \\
\hline 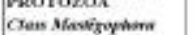 & & & & & & & & & & & & \\
\hline Evather or se & $26 \pm 22.35$ & $23 \pm 22.54$ & $27+21,49$ & $12 \pm 19.14$ & $14 \pm 17,12$ & : & 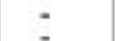 & $28 \pm 28.44$ & $11 \pm 17.97$ & - & $21 \pm 21.71$ & $9 \neq 15.73$ \\
\hline 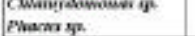 & - & 39.31 .7 & $\begin{array}{l}39+27+14 \\
32+29.27\end{array}$ & $\begin{array}{l}86+15,63 \\
16+25.46\end{array}$ & $=$ & 5 & $=$ & - & $=$ & $z$ & & \\
\hline Tetal Mautigophar & $26 \div 22.56$ & $\$ 2 .+1.0^{*}$ & $5 s+71.19$ & $33+31.67$ & $14+17,12$ & 0.9 .60 & $0 \div 0.40$ & $25<25.40$ & $11+17,7^{n}$ & $9 \div 0.40$ & $21+21.71$ & $29-28.9+$ \\
\hline Caves Sarrededina & & & & & & & & & & & & \\
\hline Anrifa dirrabies & $20+57.96$ & $93+23.61$ & $7+46.69$ & $96 \div 28.14$ & $26 \div 29.73$ & - & $5+10.40$ & $99+57,69$ & $21+15.93$ & $65+65.46$ & $n+35.7$ & $29+22.97$ \\
\hline 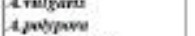 & $8+1+72$ & $\begin{aligned} 33 & \pm .39 .13 \\
3 & \pm 6.58\end{aligned}$ & $\begin{array}{l}78+5.72 \\
84 \pm 13699\end{array}$ & $93 \pm 20.24$ & $=$ & $10 \pm 1$ & $27 \pm 11.24$ & $+6=4$ & $29 \pm 11$ & $13 \pm 21.45$ & 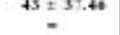 & $30=5.74$ \\
\hline Amorgenteace & - & $6.5+10.58$ & $k 3+k 4,1^{-}$ & $47+26.67$ & - & - & - & $27+28$ & $22+10$ & - & & \\
\hline CAntiest & $139+62.75$ & $154+51.59$ & $118+118.46$ & $4+10.54$ & - & $8+16.78$ & $15+16.52$ & - & - & - & $21+34.76$ & $13+19,-4$ \\
\hline Amerilat ing & $0 \pm 0.40$ & $39 \pm 67.77$ & $17 \pm 32.44$ & $8 \pm 21.63$ & & & & & & & & \\
\hline Yoeal Arertha & $226=96.08$ & $f^{7} 1=146.47$ & $f 25 \pm 2+6.51$ & $221 \pm 356^{\circ}$ & $26 \pm 20.72$ & $18 \pm 21.11$ & $92=21.54$ & $122 \pm \pm 9.49$ & $72 \pm 21.16$ & $139 \pm 6.98$ & $156 \pm 6.52$ & $83=36.22$ \\
\hline 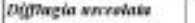 & $4+21.92$ & $53+28.7$ & $57+37.44$ & $52+22.52$ & $55+36.55$ & $16+15.35$ & $3 n+30.15$ & $77 \div 48.22$ & & & $35+20.35$ & \\
\hline Dactuminest & $s 1=19.09$ & $4=28.85$ & $89 \pm 61.65$ & $64 \pm 15.86$ & $72 \pm 25.16$ & $9=14 a b$ & $28 \pm 25.45$ & $48 \pm 41.56$ & $72 \pm 26.45$ & $6 \pm 15.91$ & $37 \pm 33.74$ & $y=2 \%, 4$ \\
\hline Dablawesv & $12 \pm 13.18$ & $37 \pm 13,49$ & $133 \pm 112,46$ & $65 \pm 36.62$ & $34 \pm 29.59$ & $31 \pm 1271$ & $s e \pm 3426$ & $33 \pm 16.11$ & $48 \pm 9,64$ & - & $35 \pm 36.64$ & $21 \pm 16.96$ \\
\hline tomowir & $14 \div 13.44$ & 4014.63 & & - & $21+40.80$ & $x+13.56$ & $35=30.65$ & $41+21.34$ & - & - & $44 \div 54.46$ & $12+22.56$ \\
\hline Wules & $31 \pm \$ 2.0^{7}$ & $4=10.57$ & $18 \pm 52.83$ & $28+35.71$ & $16 \pm 21.65$ & - & - & $19 \pm 13.24$ & $28 \pm 15,97$ & - & $37 \pm 21.14$ & $18 \pm 15.3 t$ \\
\hline Аламенте & & $6=11.66$ & & & - & - & - & $21 \pm 26.91$ & $75 \pm 3$ & - & - & $19 \pm 17.97$ \\
\hline 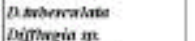 & $5 \div 26.27$ & $:$ & : & $38+8.66$ & $z$ & $=$ & z & $\therefore$ & - & - & = & $26+21.20$ \\
\hline 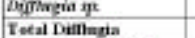 & $149+51.59$ & $171+54.44$ & $274+149.15$ & $24^{\circ}+4.17$ & $198+54.64$ & $64+30.36$ & $160+57.24$ & $\frac{12+10.41}{214+-4.14}$ & 16126.89 & $19+48.85$ & - & $8+15.46$ \\
\hline 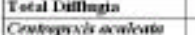 & & & & & & $64+3036$ & & $214+-3.35$ & 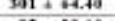 & $19+28.95$ & $191+65.62$ & $161+41.45$ \\
\hline 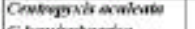 & $63+2 \mathrm{~B} 3 \mathrm{si}$ & $54+21.12$ & $193+130.27$ & $38+22,6^{-6}$ & $31+33.24$ & $26+37.87$ & $6+26.96$ & $19+28,66$ & $35+33.19$ & $57+51.98$ & $96 \div 61.68$ & $160+66.14$ \\
\hline CAnumiphumira & $+0 \div 14.50$ & $32 \ldots .65 s$ & $\mathbf{s s t}+7 \mathbf{s . 9 6}$ & $93+45+3$ & $40+35.67$ & - & & $41+52.01$ & $23+29.12$ & $73+15$ & $35+27.45$ & $26+15.54$ \\
\hline Emmiti & $2 \pm 40.12$ & $4 \pm=21.43$ & $48 \pm 52.59$ & $52 \pm 99.62$ & $46 \pm 34.99$ & $18=19.92$ & $y 9 \pm 42.96$ & $99=58.13$ & $93 \pm 24.34$ & & $s:=09.59$ & $+5=39,35$ \\
\hline Convimats & $250 \pm 93.28$ & $29 \pm 24.43$ & $68 \pm 64.39$ & $4 \pm \pm 28.74$ & - & $20 \pm 24.38$ & $46 \pm 46.29$ & $47 \pm 38.71$ & $126 \pm .4$ & $51 \pm 34-7$ & $S 2 \pm 24.46$ & $36 \pm 13.89$. \\
\hline Crithiats & $165+-7 \times 04$ & 60.94 & $16 \div 28.44$ & $t+13,84$ & $32+19,74$ & & & & & & & \\
\hline Cosspgan in & - & & & $8 \pm 1+.14$ & $27 \pm 29.54$ & $13=27.95$ & $12=41.28$ & - & $5 s= \pm 1.25$ & - & $3 \pm=33.49$ & $f E \pm 28.92$ \\
\hline Toeal Centapyodis & $532+139.22$ & $195 \div+1.9^{7}$ & $320+204.97$ & $229+42.6^{7}$ & $226+7.41$ & $71+7.32$ & $161+6.31$ & $236+63.53$ & $580+56.4$ & $181+67.52$ & $271+7.59$ & $272+111.55$ \\
\hline L A & $26+11.77$ & 40.9 .4 & $18+22,64$ & - & - & $21+31.45$ & $37+26.98$ & - & & & & - \\
\hline Luavirnta & $21 \div 15.08$ & $8+11.7$ & - & & $21+15.44$ & $25+757$ & - & $z$ & $50+62.21$ & $20+7.79$ & $21+22.44$ & - \\
\hline 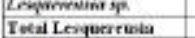 & $f+22.59$ & $\frac{11 \pm 16.66}{25 \pm 21.65}$ & $27 \pm 3.64$ & $\frac{21 \pm 33.4}{36 \pm 33.12}$ & $21 \pm 15.4$ & $46 \pm 31.91$ & $37 \pm 26.05$ & - & $39 \pm 0.21$ & $26 \pm 7.99$ & $21 \pm 2 x^{2}+4$ & - \\
\hline Eughpiar & & $37 * 19.65$ & $3 s+57.45$ & $35+3.65$ & $53+40.25$ & $15+17.56$ & $4+57.90$ & $124+5.51$ & $32+24.41$ & $\cos +19.41$ & $11+39.23$ & $22+23.71$ \\
\hline 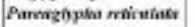 & - & & - & - & - & - & - & - & - & - & - & $16 \pm 18.76$ \\
\hline 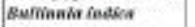 & - & - & $23 \pm 43.54$ & $16 \pm 21.22$ & - & $15 \pm 18.33$ & $16=19.64$ & $20 \pm 3$ & $=$ & - & - & - \\
\hline Plagiegsont habline & $2+34.33$ & 12.7 & & $8+14.67$ & 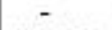 & & - & 26 & $33+39$ & - & - & $3+15.95$ \\
\hline 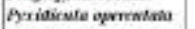 & - & $22 \pm 21.7$ & $32+71.62$ & $23 \pm 31.64$ & $37 \pm 22 . \%$ & $21 \pm 25.79$ & - & 3 & & - & - & - \\
\hline Fibera flaberinsiew & - & \pm 16.66 & $8 \pm 29.1$ & - & - & $19 \pm 15.69$ & $28 \pm 17.84$ & 15 & - & - & 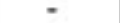 & - \\
\hline Arthau reblo ratiose & - & ess & & - & $21+25,45$ & - & & $11+180$ & 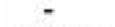 & - & $21+3.46$ & $7+11.75$ \\
\hline 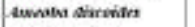 & - & $4+8.78$ & $9+31.4^{4}$ & - & $6+15.78$ & - & - & $21+18.26$ & $21+29.44$ & - & $15 \div 24.62$ & $15+21.65$ \\
\hline 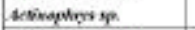 & - & $6 \pm 14.94$ & $23 \pm \$ 5.82$ & - & & $9 \pm 2187$ & - & - & - & - & & $6 \pm 11.86$ \\
\hline Tueal Swevoritas & $977 \pm 250.78$ & $9: 4 \pm 195.14$ & $201 \pm 558.54$ & $815 \pm 13520$ & $508 \pm 15316$ & $282 \pm 112.65$ & $958=224.7$ & $820 \pm 235.97$ & $958 \pm 145.99$ & $431 \pm 114.11$ & $716 \pm 184.41$ & $553 \pm 167.55$ \\
\hline Gas CW: & & & & & & & & & & & & \\
\hline cambintum & - & $39 \pm 34.65$ & $125 \pm 127,42$ & $112 \pm 60.19$ & - & $9=11.95$ & $21= \pm t$ & $\$ 4=26.53$ & $145 \pm$ & $52 \pm 4$ & $149 \pm 59.85$ & $29=29.69$ \\
\hline & - & & & & & & & & & & & \\
\hline & - & . & & & - & - & & - & - & 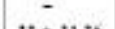 & a & \\
\hline 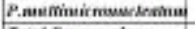 & $42 \pm 2+.58$ & $t 9=17.94$ & & & $3^{57} \pm+0.70$ & $19=14.7$ & $50=20.51$ & $45 \pm 21.5^{*}$ & $59 \pm 28.52$ & $3 s=31.26$ & $7=30.64$ & $39 \pm \pm 9.62$ \\
\hline Tasti Parramerianas & $42 \pm 2+38$ & $I 21=51.22$ & $299 \pm 215.58$ & & $35 \pm+2.79$ & $27 \pm 12.94$ & $57 \pm 26.95$ & $9 \pm 31.83$ & $284 \pm 81.56$ & $25 \pm 53.4^{7}$ & $153=5574$ & $6 s \pm 42.25$ \\
\hline & - & $12 \cdot 1$ & & & & & & & & & & \\
\hline in & - & 43. & & & & & & & & & & $+30.6 \%$ \\
\hline & - & & $177 \pm 75,96$ & & 32 & $19=2$ & $16 \pm 21.35$ & & & & & $23 \pm 21.86$ \\
\hline & - & $s \cdot 13$ & & & $26+31.19$ & - & - & & & & & - \\
\hline & - & & 15.83 & & & - & - & & & & & - \\
\hline 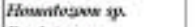 & - & $=18.56$ & & & - & - & - & & 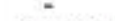 & $=$ & • & - \\
\hline & - & 73 & & & $36 \pm 18.91$ & - & - & & & & $52 \pm 23,4$ & $29 \pm 2$ \\
\hline spleara: & - & $35 \div 30.54$ & & & & $15-35.39$ & $21+24.59$ & $31+31.51$ & as $\mathrm{a}$ & & $4.3 \div 1+.23$ & - \\
\hline wat sp & - & $4=19$ & & & 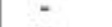 & & & & & & & - \\
\hline 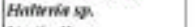 & - & & \pm 3 & $\pm \mathbf{8 . 8 8}$ & - & - & - & - & $=$ & & 38.39 & $65 \pm 25.97$ \\
\hline Sartirnus & $z$ & $14 \div-14.46$ & $194+28,31$ & $30+65.08$ & inst & -8 & & $86 \div 28$ & $53+32.21$ & 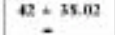 & $26+32.82$ & $05 \div-46.61$ \\
\hline 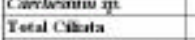 & $2+24.5 s$ & $208+100.5 s$ & $1240+259.12$ & 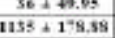 & 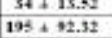 & $152+4^{-9}-96$ & $\begin{array}{l}59+24.31 \\
200+57.4^{*}\end{array}$ & $522+67.6$ & & $16+114.45$ & $\begin{array}{l}36+11.89 \\
44+6.55\end{array}$ & $564+92.93$ \\
\hline & & & $2538 \pm 735.40$ & & & $414 \pm 125.49$ & $759 \pm 155.58$ & $11 \% 0 \pm 91.11$ & $92 \pm 188.05$ & $650 \pm 209.45$ & $1331=236.71$ & $91^{*} \pm 234.42$ \\
\hline
\end{tabular}

$18 \pm 31.55 \mathrm{n} / \mathrm{l}$ ) and Bullinula (nil to $18 \pm 28.83$ ) and > Actinophrys (nil to $12 \pm 30.82 \mathrm{n} / \mathrm{l}$ ). Quantitatively, during the subsequent year viz. 2003-04, the order of dominance of various genera belonging to Class Sarcodina has been noticed as Arcella (18 21.11 to $471 \pm 106.47 \mathrm{n} / 1)>$ Centropyxis $(71 \pm 74.32$ to $380 \pm 96.47 \mathrm{n} / 1)>$ Difflugia $(19 \pm 28.88$ to $301 \pm 64.40 \mathrm{n} / \mathrm{l})>$ Euglypha (nil to $124 \pm 88.51 \mathrm{n} /$ 1) $>$ Lesquereusia (nil to $47 \pm 22.59 \mathrm{n} / \mathrm{l}$ ) > Plagiopyxis (nil to $42 \pm 34.33 \mathrm{n} / \mathrm{l}$ ) > Pyxidicula (nil to $37 \pm 22.76 \mathrm{n} / \mathrm{l}$ ) $>$ Nebela (nil to $28 \pm 17.84 \mathrm{n} / \mathrm{l})>>$ Bullinula (nil to $23 \pm 43.90 \mathrm{n} / \mathrm{l}$ ) and Actinophrys (nil to $23 \pm 35.82 \mathrm{n} / \mathrm{l}$ ) $>$ Amoeba (nil to $21 \pm 28.26 \mathrm{n} / \mathrm{l}$ ) and Astramoeba (nil to $21 \pm 25.48 \mathrm{n} / \mathrm{l}$ ) and > Pareuglypha (nil to $16 \pm 27.22 \mathrm{n} / \mathrm{l}$ ).

Class Sarcodina, during the first year, showed bimodal viz. April and August peak, with a trough during July and December. It observed April highest and December lowest record(Table 1 and Fig. 1b). In the subsequent year, class Sarcodina observed an increase during April, October and December and trough during July and November. It recorded highest and lowest record during April and July, respectively (Table 2 and Fig.1b).

Among class Ciliata, during the first year, the order of quantitative dominance of various genera has been observed as Paramecium (nil to 133 $\pm 43.93 \mathrm{n} / \mathrm{l}$ ) > Euplotes (nil to $92 \pm 31.42 \mathrm{n} / \mathrm{l}$ ) > Coleps (nil to $56 \pm 49.45 \mathrm{n} / \mathrm{l}$ ) > Condylostoma (nil to $51 \pm 111.71 \mathrm{n} / \mathrm{l}$ ) $>$ Vorticella (nil to $39 \pm 70.87 \mathrm{n} / \mathrm{l})>$ Stylonychia $($ nil to $29 \pm 51.71 \mathrm{n} / \mathrm{l})>$ Uronema (nil to $24 \pm 48.48 \mathrm{n} / \mathrm{l}$ ) and Carchesium (nil to $24 \pm 33.82 \mathrm{n} / \mathrm{l}$ ) $>$ Urocentrum (nil to $23 \pm 39.21 \mathrm{n} / \mathrm{l}$ ) > Halteria (nil to $22 \pm 21.38 \mathrm{n} / \mathrm{l}$ ) > Homalozoon (nil to $17 \pm 43.18 \mathrm{n} / \mathrm{l}$ ) and > Lionotus (nil to $11 \pm 10.14 \mathrm{n} / \mathrm{l}$ ). The order of quantitative dominance of various genera of class ciliata, during the 
subsequent year, has been noticed as Paramecium $(37 \pm 40.70$ to $299 \pm 215.58 \mathrm{n} / \mathrm{l})>$ Coleps (nil to $177 \pm 75.96 \mathrm{n} / \mathrm{l})$ $>$ Euplotes (nil to $168 \pm n / l)>$ Uronema (nil to $135 \pm n / l)>$ Halteria (nil to $129 \pm \mathrm{n} / \mathrm{l})>$ Vorticella (nil to $104 \pm 35.31 \mathrm{n} / \mathrm{l}$ ) $>$ Lionotus (nil to $98 \pm 39.76 \mathrm{n} / \mathrm{l}$ ) > Urocentrum (nil to $94 \pm 104.11 \mathrm{n} / \mathrm{l})>$ Homalozoon (nil to $79 \pm 27.23 \mathrm{n} /$ 1)>Condylostoma (nil to $74 \pm 19.47 \mathrm{n} / 1$ ) $>$ Stylonychia (nil to $68 \pm 30.65 \mathrm{n} / \mathrm{l}$ ) and Carchesium (nil to $68 \pm 15.85 \mathrm{n} / \mathrm{l}$ ) and $>$ Chilodonella (nil to32 $\pm 39.45 \mathrm{n} / \mathrm{l}$ ).

Class Ciliata, during the first year, recorded summer (March, April and May) quantitative increase with constant decrease during November, December and January. These recorded highest and lowest record during April and December, respectively (Table 1 and Fig. 1c). In the subsequent year, ciliates showed April, October and December peaks. These observed April highest and February lowest record (Table 2 and Fig. 1c). Seasonally, total protozoans, have shown April and August peak and December and January trough, during the first year. These recorded April highest and December lowest quantitative count (Table 1 and Fig. 1d). In the subsequent year, protozoans recorded trimodal viz. April, October and December peaks with April highest and July lowest record. These recorded July and November trough (Table 2 and Fig. 1d).

Summer (March-May), qualitative and quantitative increase of protozoans, during both the years of study, is in accordance with the findings of Chourasia and Adoni (1985), Dutta etal.(1990,1991), Katiyar and Belsare (1997), Wetzel(2000) and Narayana and Somashekhar (2002) and may be attributed to- i) increased multiplication at higher temperature (Sehgal, 1980; Kumar, 1990; Katiyar, 1995; Katiyar and Balsare, 1997; Dutta and Sharma, 2000; Sharma, 2000, and Narayana and Somashekhar, 2002), ii) rise in $\mathrm{pH}$. A direct relationship between protozoan density and $\mathrm{pH}$ is already on record ( Rai and Sharma,1986). iii) enrichment of organic matter and dissolved solids due to accelerated decomposition of aquatic vegetation (Kudo,1986; Kaushik and Saksena,1995; Katiyar and Belsare,1997; Pandit, 1999 and Wetzel, 2000), at higher temperature, iv) summer upward migration of some rhizopod protozoans due to fat inclusions(Wetzel, 2000 and Hutchinson, 2004) and gas bubbles(Cole, 1975; Reid and Wood, 1976; Wetzel, 2000 and Hutchinson , 2004).

Dilution, depletion of food and increased turbulence, during monsoon, may account for July low protozoan count in lake water, during both the years of study and is in conformity with the observations of Vashisht and Jindal(1980), Jindal and Vasisht(1985), Dutta et al.(1990 and 1991) and Narayana and Somasekhar (2002).

Winter viz., December, January/November and January low quantitative count of protozoans, during the year 2002-03/2003-04, has its correlation with- i) winter overturn which results in uniform mixing of protozoans and hence their reduction in the surface waters, ii) presence of low concentration of DO and rise in free $\mathrm{CO}_{2}$ (Jindal and Vasisht, 1985 and Dutta et al., 1990). iii) sinking of some protozoans (Difflugia) to the bottom layers due to metabolization of fat gloubulets during winter (Wetzel, 2000).

Ecological distribution of protozoans has been discussed earlier by many workers. Chourasia and Adoni(1985), Mishra and Saksena(1990), Sleigh et al.(1992) and Pandit(2000) have recorded a positive correlation between temperature and protozoans. Jindal and Vasisht(1985) and Patra and Dutta(2004) correlated protozoans abundance with moderate temperature and higher record of DO. Rai and Sharma(1986) worked out positive correlation between the total protozoan count and chemical factors like $\mathrm{pH}$, DO, bicarbonate, alkalinity etc. Dutta et al.(1990) found rise in temperature, high value of $\mathrm{pH}$, DO, calcium, and total hardness favourable for quantitative rise in protozoans in some pools adjacent to river Tawi, Jammu. Dutta et al. (1991) worked out rise in temperature, rise in $\mathrm{pH}$, low bicarbonate, calcium, magnesium and total hardness favouring protozoan abundance in Kunjwani pond, Jammu. Narayana and Somashekhar(2002) reported positive correlation between protozoans and temperature, phosphates and nitrates. Katiyar and Belsare(1997) recorded positive correlation between total protozoans and nitrate and phosphate, during summers, from Bhopal lakes.

Table 3. Coefficient of correlation( $r$ ) between Protozoans and various physico-chemical parameters of Surinsar Lake, Jammu (Feb. 2002-Jan. 2004).

\begin{tabular}{lcc}
\hline Parameters & $(\mathbf{r}) \mathbf{2 0 0 2 - 0 3}$ & $(\mathbf{r}) \mathbf{2 0 0 3 - 0 4}$ \\
\hline Water Temperature & 0.22 & -0.25 \\
Depth & 0.07 & -0.03 \\
Turbidity & 0.74 & 0.10 \\
Salinity & 0.04 & 0.23 \\
Electric Conductivity & 0.06 & 0.03 \\
TDS & -0.03 & 0.11 \\
pH & 0.32 & -0.42 \\
Free $\mathrm{CO}_{2}$ & 0.63 & 0.04 \\
Dissolved $\mathrm{Oxygen}^{2-}$ & -0.54 & 0.22 \\
Carbonate $\left(\mathrm{CO}_{3}{ }^{2-}\right)$ & -0.36 & 0.29 \\
Bicarbonate $\left(\mathrm{HCO}_{3}{ }^{-}\right)$ & -0.36 & 0.23 \\
Chloride $\left(\mathrm{Cl}^{-}\right)$ & -0.36 & -0.02 \\
Calcium $\left(\mathrm{Ca}^{2+}\right)$ & -0.31 & -0.09 \\
Magnesium $\left(\mathrm{Mg}^{2+}\right)$ & 0.10 & -0.24 \\
Total $\mathrm{Hardness}$ & 0.22 & 0.37 \\
BOD & -0.54 & 0.49 \\
COD & 0.75 & -0.32 \\
Sodium $\left(\mathrm{Na}^{+}\right)$ & 0.15 & 0.51 \\
Potassium $\left(\mathrm{K}^{+}\right)$ & 0.03 & -0.29 \\
Phosphate $\left(\mathrm{PO}_{4}{ }^{3-}\right)$ & -0.62 & 0.09 \\
Nitrate $\left(\mathrm{NO}_{3}\right)$ & 0.59 & -0.35 \\
Sulphate $\left(\mathrm{SO}_{4}{ }^{2-}\right)$ & -0.13 & -0.10 \\
Silicate $\left(\mathrm{SiO}_{2}\right)$ & -0.18 & -0.38 \\
Iron $\left(\mathrm{Fe}{ }^{2+}\right)$ & -0.28 & -0.04 \\
\hline
\end{tabular}


Table 4. ANOVA test for various Classes of Protozoans ( Feb. 2002-Jan. 2004).

\begin{tabular}{|c|c|c|c|c|c|c|}
\hline Groups & Count & & Sum & & Average & Variance \\
\hline Total Mastigophora & 12 & & 317 & & 26.42 & 1158.63 \\
\hline Total Sarcodina & 12 & & 4737 & & 394.75 & 83957.30 \\
\hline Total Ciliata & 12 & & 1624 & & 135.33 & 12305.70 \\
\hline \multicolumn{7}{|l|}{ ANOVA } \\
\hline $\begin{array}{l}\text { Source of } \\
\text { Variation }\end{array}$ & SS & df & & MS & $\mathbf{F}_{\text {obs }}$ & $\mathbf{F}_{\text {crit }}$ \\
\hline Between Groups & 859317.17 & 2 & & 429658.583 & 13.23 & 3.28 \\
\hline Within Groups & 1071637.83 & 33 & & 32473.8737 & & \\
\hline Total & 1930955 & 35 & & & & \\
\hline \multicolumn{7}{|c|}{ (Feb 2003-Jan 2004) } \\
\hline Groups & Count & & Sum & & Average & Variance \\
\hline Total Mastigophora & 12 & & 348.04 & & 29.00 & 917.07 \\
\hline Total Sarcodina & 12 & & 8827.56 & & 735.63 & 69541.71 \\
\hline Total Ciliata & 12 & & 5468.67 & & 455.72 & 143455.65 \\
\hline \multicolumn{7}{|l|}{ ANOVA } \\
\hline $\begin{array}{l}\text { Source of } \\
\text { Variation }\end{array}$ & SS & df & & MS & $\mathbf{F}_{\text {obs }}$ & $\mathbf{F}_{\text {crit }}$ \\
\hline Between Groups & 3039033.60 & 2 & & 1519516.80 & 21.31 & 3.28 \\
\hline Within Groups & 2353058.80 & 33 & & 71304.81 & & \\
\hline Total & 5392092.40 & 35 & & & & \\
\hline
\end{tabular}

Where, $\mathrm{SS}=$ Sum of Squares, $\mathrm{df}=$ degree of freedom, MS= Mean Square(SS/df), Fobs= observed value of F, Fcrit= critical value obtained from the $\mathrm{F}$ distribution table at $5 \%$ level.
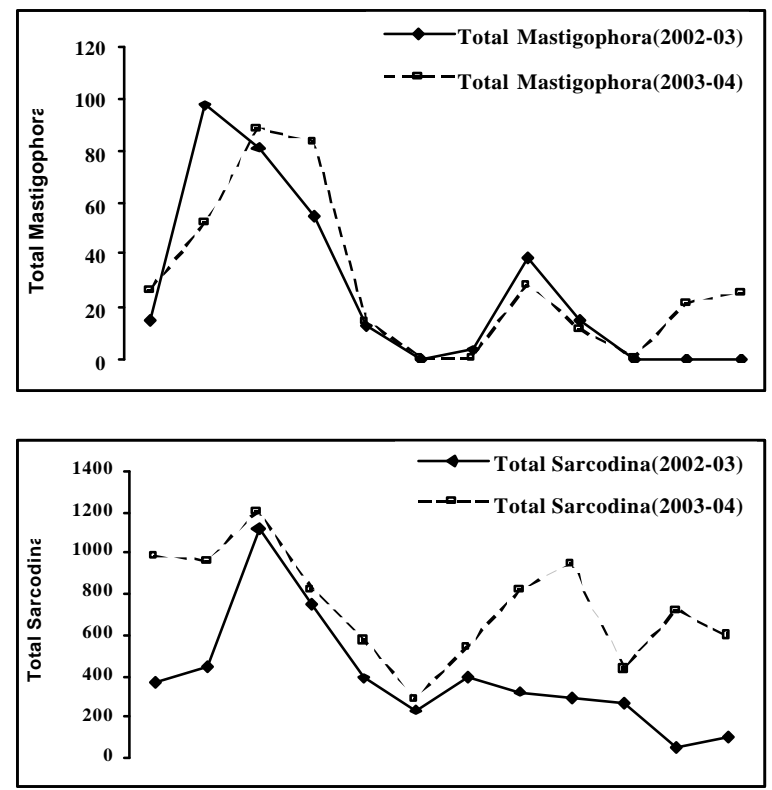
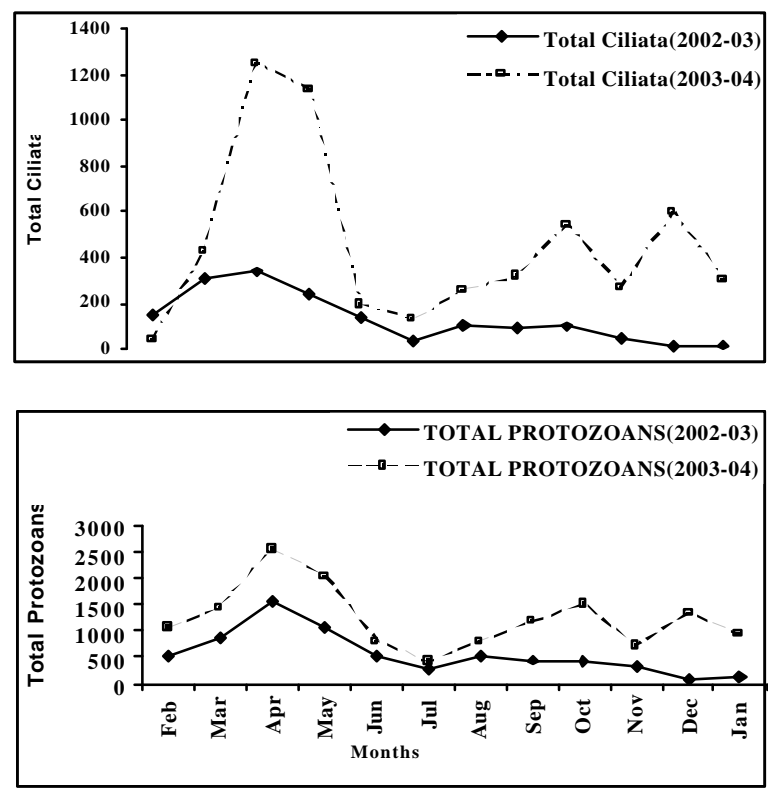

Fig. 1. Mean monthly variations in total mastigophora, total sarcodina, total ciliata and total protozoa of Surinsar Lake, Jammu.

Analysis of coefficient of correlation of protozoans, with different physico-chemical parameters of Surinsar Lake water has shown significant results with $\mathrm{pH}$, free $\mathrm{CO}_{2}$, carbonate, BOD, COD, phosphate and nitrate only, during the year 2002-03. In the subsequent year viz. 2003-04, coefficient of correlation analysis ( $r$ ) of total protozoan with various physico-chemical factors of water has generally revealed insignificant correlation except with sodium(Table 3). This strongly indicates that no single factor is a strong determinant for protozoan diversity and density in lake Surinsar, Jammu. Also, one way ANOVA analysis of the means of all the three Classes of protozoans shows that because the calculated value of $\mathrm{F}$ is greater than the tabulated value of $\mathrm{F}$ at $\mathrm{p}=0.5(3.28)$, the calculated value is significant and the means of various classes differ significantly among themselves(Table 4). Thus, all these classes are significant contributor to the total protozoan population. 


\section{ACKNOWLEDGEMENTS}

Sincere thanks are due to Head, Department of Environmental Sciences, for laboratory and other facilities. Financial assistance provided by Jammu University, Jammu is greatly acknowledged.

\section{REFERENCES}

Cairns, J. Jr. and McCormick, P. V. (1993). Handbook of hazardous materials (pp. 627-638). New York: Academic Press.

Chourasia, S.K. and Adoni, A.K. (1985). Zooplankton dynamics in a Shallow entropic lake. In: A.D. Adoni(Ed.). Proc. Nat. Symp. Pure and Applied Limnology Bull. Bot. Soc. Sagar, 32:30-39.

Cole, G. A. (1975). A text book of Limnology. The G. V. Mosby Company. Saint Louis.

Dutta, S.P.S., Kumar, S. and Kumari, V. (1991). Seasonal fluctuations in protozoans in Kunjwani pond, Jammu. Geobios New Reports, 10(2): 121-124.

Dutta, S.P.S. and Sharma, J. (2000). Ecology of zooplankton of sewage fed Farooq Nagar pond,Jammu. $J$. Natcon.,12(1):71-78.

Dutta,S.P.S.(1983). On some freshwater rhizopoda from Jammu. Part -I, Review Science, 1:91 -94.

Dutta,S.P.S., Zutshi,N. and Puri, A. (1990). Ecology of protozoans from some pools present along the sides of the River Tawi, Near Nagrota, Jammu. J. Hydrobiol., 6 (1):19-24.

Edmondson, W.T. (1992). Freshwater biology. John Wiley and Sons, Inc., New York:1248pp.

Hutchinson,G.E.(2004). A treatise on Limnology. Vol. IIIntroduction to lake biology and the limnoplankton.John Wiley and Sons, Inc., New York.

Jhingran,V.J.(1991). Fish and fisheries of India. Hindustan Publ. House, India.

Jiang, J. G. and Shen, Y. F. (2007). Development of the microbial communities in Lake Donghu in relation to water quality. Environ. Monit. Assess., 127:227-236.

Jindal, R. and Vasisht, H.S. (1985). Ecology of freshwater pond at Nabha. Zoologica Orientalis, 2(1-2): 74-83.

Katiyar, S.K. (1995). Limnological studies of Bhopal lakesI: Protozoa fauna of polymictic \& homothermal lake. $J$. Freshwat. Biol., 7(3):159-163.

Katiyar, S.K and Belsare, D.K.(1997). Limnological Studies on Bhopal Lakes: Fresh water protozoan communities as indicators of organic pollution .J. Environ. Biol., 18(3): 271-282.

Kaushik, S. and Saksena, D. N. (1995) . Trophic status rotifer fauna of certain water bodies in Central India. J. Environ. Biol., 16 : 283-291.

Khan, I.L. and Khanam, A. (2004). Fundamentals of Biostatics. Ukaaz Publ., Hyderabad.pp330:336.

Kotangale,J.P.(1995). Zooplankton Ecology of Sewage Fertilized Fish Pond. J. Ecotoxicol. Environ. Monit., 5(4):241-248.

Kudo,R.R.(1986). Protozoology published by Books and Periodical Corporation (India):1174pp.

Kumar, S.(1990). Limnology of Kunjwani Pond, Jammu ,with special reference to Plankton and
Macrophytes.M.Phil. Dissertation submitted to University of Jammu, Jammu.

Lee, S., Basu, S., Tyler, C.W., andWei, I.W. (2004). Ciliate populations as bio-indicators at Deer Island Treatment Plant. Advances in Environmental Research, 8, 371-378. Lugo, A., Alcocer, J., Sanchez, M. R., and Escobar, E. (1998). Littoral

Mishra,S.R.and Saksena,D.N.(1990). Seasonal abundance of zooplankton of waste water from the industrial complex at Birla Nagar(Gwalior) India.Acta. Hydrochim. Hydrobiol., 18:215-220.

Naidu, K.V.(1966). Studies on the fresh water protozoa of South India III. Euglenoidina - 2. Hydrobiolgia., 27(1-2): 23-32.

Nair,K.N., Das,A.K. and Mukherjee,B.N.(1971.). On some freshwater Rhizopoda and Heliozoa(Protozoa) from Kolkata and its Environs.Part I. Res. Zool. Surv. India, 65(1-40):1-6.

Narayana, J. and Somashekar(2002).Distribution Ecology of Protozoa in Relation to Water quality in River Cauvery, Karnatka, India. Ecology and Ethology of Aquatic Biota. Vol.I. Daya Publ. House, New Delhi.pp 283-293.

Pandit, A.K.(1999). Trophic structure of plankton community in some typical wetlands of Kashmir, India. In. Limnological Research in India. Ed. by S.R.,Mishra. pp190-224.

Pandit, A.K.(2000). Plankton as Indicators of Trophic Status of Wetlands. Ecology and Ethology of Aquatic Biota. Daya Publ.House,New Delhi. Vol. I.pp 341-360.

Patra, S.B. and Datta, N.C. (2004). Seasonal fluctuation of different zooplanktonic groups of a rainfed wetland in relation to some abiotic factors. Ind. J. Environ. and Ecoplan., 8(1):07-12.

Rai, D.N. and Sharma,U.P. (1986). Studies on theoccurrence of periphytic Testacea(Protozoa: Rhizopoda) in the weed infested pond of Dharbhanga(North Bihar). Ind. J. Ecol.,13(2):339-334.

Reid, G.K. and Wood, R.D.(1976). Ecology of Inland waters and esturies. D. Van Norstand Company, New York:485 $\mathrm{pp}$.

Sehgal H.S. (1980). Limnology of Lake Surinsar, Jammu, with reference to Zooplankton and fishery prospects. Ph.D. Thesis submitted to Deptt. of Biosciences. University of Jammu.

Sharma, M.(2000). Ecology and community structure of Zooplankton of Mansar lake ,Jammu. Ph.D. Thesis submitted to University of Jammu, Jammu.

Sharma, R.K. and Rathore,V. (2000). Pollution Ecology with reference to Commercially important Fisheries prospect in a Rural Based Waterbody: The Lake Sarsai Nawar, Etawah in U.P.(India). Poll. Res., 19(4): 641-644.

Sleigh, M. A., Baldock,B.M.and Baker, J.H.(1992). Protozoan communities in Chalkstreams. Hydrobiologia, 248:53-64.

Srivastava,K.N.,Srivastava,P.andSinha,A.K.(1990). Zooplankton studies of Ganga river between Kalakanker (Pratapgarh) and Phaphmau (Allahabad) U.P.In: Recent Trends in limnology. Ed. by Agarwal,V.P. and Das,P. Jagmander Book Agency:129 -133

Trivedy, R.K., Goel, P.K. and Trisal,C.L (1987). Practical Methods in Ecology and Environmental sciences. 
Environmental Publications, Post office 60, Karad.

Vasisht, H.S. and Jindal,R. (1980). Rheological survey of a Pucka stream at Patiala, Punjab(India). Limnol. (Berlin), 12 (1) : 77-83

Verma, S.R. and Dalela, R.C.(1974). Studies on the pollution of Kali Nadiby Industrial wastes near Mansurpur,Part II: Biological Index of Pollution and biological characteristics of the river. Acta. Hydrochin.Hydrobiol.,3:259-274.

Welch, P.C.(1948). Limnological methods, McGraw Hill Book Co.Inc. New York.

Wetzel, R.G.(2000). Limnology. Lake and River Ecosystems.
Third Ed.Academic Press, London.

Xu, K., Choi, J. K., Yang, E. J., Lee, K. C. and Lei, Y. (2002). Biomonitoring of coastal pollution status using protozoan communities with a modified PFU method. Marine Pollution Bulletin, 44: 877-886. 